\title{
Philosophiques
}

\section{Les élections sont-elles essentielles à la démocratie ?}

\section{Hervé Pourtois}

Volume 43, numéro 2, automne 2016

URI : https://id.erudit.org/iderudit/1038213ar

DOI : https://doi.org/10.7202/1038213ar

Aller au sommaire du numéro

\section{Éditeur(s)}

Société de philosophie du Québec

ISSN

0316-2923 (imprimé)

1492-1391 (numérique)

Découvrir la revue

Citer cet article

Pourtois, H. (2016). Les élections sont-elles essentielles à la démocratie? Philosophiques, 43(2), 411-439. https://doi.org/10.7202/1038213ar

\section{Résumé de l'article}

En dépit de débats nourris sur la délibération et la représentation démocratiques, la question de la justification de l'élection comme mode de désignation des gouvernants a été peu abordée par la philosophie politique contemporaine. Cette question est pourtant importante. Une confrontation avec l'alternative que pourrait constituer le tirage au sort d'une assemblée représentative permet d'identifier les vertus spécifiques de l'élection au regard de quatre critères de légitimité démocratique : le consentement et la responsabilité des gouvernés, l'inclusion égalitaire des citoyens, le contrôle des décideurs et la contestabilité des décisions, la qualité épistémique de la décision. L'analyse conduit à conclure au caractère essentiel de l'élection dans des démocraties de masse. C'est à partir d'elle, et non en dépit d'elle, que doit être surmontée la crise de la représentation. 


\title{
Les élections sont-elles essentielles à la démocratie?
}

\author{
HERVÉ POURTOIS \\ Chaire Hoover d'éthique économique et sociale \\ Institut supérieur de philosophie \\ Université catholique de Louvain \\ herve.pourtois@uclouvain.be
}

\begin{abstract}
RÉSUMÉ. - En dépit de débats nourris sur la délibération et la représentation démocratiques, la question de la justification de l'élection comme mode de désignation des gouvernants a été peu abordée par la philosophie politique contemporaine. Cette question est pourtant importante. Une confrontation avec l'alternative que pourrait constituer le tirage au sort d'une assemblée représentative permet d'identifier les vertus spécifiques de l'élection au regard de quatre critères de légitimité démocratique: le consentement et la responsabilité des gouvernés, l'inclusion égalitaire des citoyens, le contrôle des décideurs et la contestabilité des décisions, la qualité épistémique de la décision. L'analyse conduit à conclure au caractère essentiel de l'élection dans des démocraties de masse. C'est à partir d'elle, et non en dépit d'elle, que doit être surmontée la crise de la représentation.
\end{abstract}

L'organisation régulière d'élections libres est le critère le plus souvent utilisé, à l'échelle internationale, pour évaluer le caractère démocratique d'un régime politique (PNUD, 2005) ${ }^{1}$. L'inhérence des élections à la démocratie est du reste clairement affirmée par la Déclaration universelle des droits de l'homme:

La volonté du peuple est le fondement de l'autorité des pouvoirs publics; cette volonté doit s'exprimer par des élections honnêtes qui doivent avoir lieu périodiquement, au suffrage universel égal et au vote secret ou suivant une procédure équivalente assurant la liberté du vote»(Déclaration universelle des droits de l'homme, art. 21, al. 3, je souligne) $)^{2}$.

Pourtant le lien ainsi posé entre démocratie et élections n'a pas toujours été une évidence. Pour les anciens, tout comme pour les premiers penseurs modernes du gouvernement représentatif, la désignation des gouvernants

1. Des versions préliminaires de cet article ont fait l'objet de présentations à l'Université de Pavie, à l'Université Laval, à l'Université de Montréal et à l'Université catholique de Louvain. Je tiens à remercier les organisateurs et les participants à ces présentations ainsi que tout particulièrement et chaleureusement Philippe Coppens, Dominique Leydet, John Pitseys, PierreEtienne Vandamme et les deux évaluateurs anonymes de Philosophiques. Les commentaires qu'ils m'ont émis à différents stades de l'élaboration de cette recherche m'ont été très précieux. Cet article a été parachevé lors d'un séjour au Centre de recherches en éthique (CRÉ) à Montréal. Merci au CRÉ et au FNRS d'avoir rendu ce séjour possible.

2. La Cour européenne des droits de l'homme réaffirme ce lien. Voir notamment: CEDH

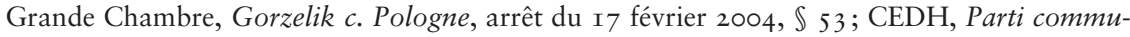
niste unifié de Turquie et autres $c$. Turquie, arrêt du 30 janvier I998, $\mathbb{S} 44$. 
par élection avait un caractère aristocratique: elle était destinée à sélectionner les meilleurs pour gouverner (Manin, I995, 93 ss.) C'est ainsi que, au début du XIX ${ }^{e}$ siècle, François Guizot pouvait encore écrire: "Le but de l'élection est évidemment d'envoyer au centre de l'État les hommes les plus capables et les plus accrédités du pays; c'est une manière de découvrir et de constituer la véritable, la légitime aristocratie» (Guizot, I 826, 395). On sait aussi que l'élection, même au suffrage égal, fut pratiquée dans des contextes et pour des raisons étrangers à la démocratie (Cristin, 20I4): pour la désignation des abbés ou des évêques, celle des recteurs ou des professeurs dans les universités médiévales et de la première modernité, etc. Enfin, pour nombre de penseurs et d'acteurs de la gauche marxiste ou anarchiste, la démocratie authentique ne pouvait reposer sur l'élection, car celle-ci n'assurait qu'une égalité et une liberté formelles, contribuant à dissimuler et à maintenir les inégalités réelles ${ }^{3}$. Cependant, dès le début $\mathrm{du} \mathrm{xx}^{\mathrm{e}}$ siècle, une bonne part de la gauche s'est ralliée au principe du suffrage universel et en a même fait une de ses revendications majeures. La droite, conservatrice ou libérale, fit de même.

Aujourd'hui, ce qui semblait ainsi être devenu une évidence normative dans la seconde moitié du $\mathrm{xx}^{\mathrm{e}}$ siècle — la démocratie suppose avant toute chose des élections libres - paraît cependant remis en question par ce que d'aucuns appellent «la crise de la représentation». Celle-ci est avant tout une crise de la force légitimante de l'élection: les enquêtes d'opinion montrent que les institutions politiques qui sont liées à l'élection ne bénéficient que d'un très faible capital de confiance ${ }^{4}$. C'est dans ce contexte que certains acteurs de la société civile ou du monde académique, parfois relayés par des autorités publiques, proposent et de temps à autre mettent en œuvre des dispositifs innovants destinés à faire participer des citoyens à des procédures délibératives à vocation consultative et quelquefois même décisionnelle en marge de la démocratie électorale. Un tel engouement pour l'innovation démocratique semble traduire une volonté de mettre la participation directe des citoyens au cœur de l'élaboration des politiques, de dépasser ainsi les insuffisances de la démocratie électorale et parfois même — quoiqu'assez rarement — de l'abolir à long terme (Van Reybrouck, 20I2). Les élections ne devraient plus jouer le rôle central qu'on a eu tendance à leur attribuer dans le processus politique et devraient être

3. «De même que les chrétiens sont égaux dans le ciel et inégaux sur la terre, les membres du peuple pris chacun dans leur singularité sont égaux dans le ciel de leur monde politique et inégaux dans l'existence terrestre de la société» (Marx I972, I35).

4. Voir notamment les données de l'Eurobaromètre relatives à «L'opinion publique dans l'Union européenne» (http://ec.europa.eu/public_opinion/archives/eb/eb82/eb82_publ_fr.pdf, consulté le io décembre 2015). Au cours des dernières années le taux de confiance moyen des Européens dans leur parlement national a oscillé entre 25 et $30 \%$. La défiance à l'égard des partis politiques tournait, quant à elle, autour de $80 \%$. 
complétées ${ }^{5}$, voire supplantées, par d'autres pratiques ou procédures participatives plus authentiquement démocratiques grâce notamment au recours à la sélection aléatoire de citoyens "ordinaires". La littérature, tant académique que militante, sur ces innovations, en particulier sur le recours au tirage au sort, est aujourd'hui extrêmement abondante ${ }^{6}$. Elle prend souvent appui sur un diagnostic critique à l'égard du fonctionnement actuel de nos systèmes représentatifs. Mais curieusement, elle n'aborde que très incidemment la question de savoir si et surtout pourquoi nous devrions tenir ou renoncer aux élections dans nos sociétés démocratiques. Penser le sens et la valeur des élections en démocratie est pourtant un défi philosophique important.

Je voudrais d'abord évoquer ce défi tout en essayant de comprendre pourquoi la question de la désignation des gouvernants ne semble guère avoir retenu l'attention des philosophes politiques récemment alors même qu'elle devrait demeurer essentielle. Dans la seconde partie de cet article, j'essaierai ensuite d'identifier quelques raisons de principe pour lesquelles des sociétés démocratiques de masse ne pourraient se passer des élections lorsqu'il s'agit de désigner leurs gouvernants. Pour ce faire, je confronterai celles-ci à l'hypothèse de leur remplacement par le tirage au sort dans le cadre de l'attribution des mandats parlementaires.

\section{La question de la désignation des gouvernants}

La question de la justification de l'élection comme mode de désignation des gouvernants est évidemment au cœur de la pensée politique des promoteurs du gouvernement représentatif. Les travaux de Bernard Manin (I995) et de Pierre Rosanvallon (I998) ont proposé des synthèses et des mises en perspective magistrales sur ces moments fondateurs. Ils ont ainsi montré que, pour ces promoteurs, le suffrage des citoyens était une condition essentielle de la légitimité du pouvoir dont disposaient les gouvernants. Mais, en dehors de ces travaux à vocation généalogique, la philosophie politique contemporaine n'a guère traité des élections dans une perspective normative ${ }^{7}$. Elle semble s'en être désintéressée, et lorsqu'elle les évoque, c'est le plus souvent pour en signaler les limites, poussant rarement la réflexion jusqu'au point de savoir si et pourquoi elles demeureraient nécessaires aujourd'hui en démocratie. On doit s'interroger sur les raisons de ce silence.

\subsection{Pourquoi un manque d'intérêt pour l'élection?}

Au cours des dernières décennies, la réflexion normative sur la démocratie s'est focalisée sur la question de la procédure de construction des décisions.

5. Voir l'idée d'un "quatrième pouvoir" proposée par Bacqué et al., 2005, p. 34 .

6. Pour une présentation synthétique de ces positions et évolutions, voir Blondiaux, 2008. Voir aussi Landemore, 20I3; McCormick, 20II ; Sintomer, 20I I et 20I2; Sutherland, 2008.

7. Relevons toutefois Thompson, 2002, et Parkinson, 2006. 
Les théories délibératives de la démocratie et les débats qui se sont noués autour de celles-ci ont été déterminants à cet égard ${ }^{8}$. Ils ont exploré la question de savoir s'il est possible et désirable d'élaborer des décisions publiques par l'échange d'arguments - plutôt que par la négociation ou la simple agrégation des préférences - et d'associer les citoyens, et pas seulement leurs représentants, à une telle délibération. Pour les tenants de l'approche délibérative, ce sont en effet les qualités participatives et argumentatives de la procédure de décision politique qui confèrent à celle-ci sa légitimité ${ }^{\text {. }} \mathrm{La}$ démocratie ne peut être réduite à un simple mécanisme de formation d'un choix politique par agrégation des préférences ou des opinions; elle suppose avant tout qu'un processus public et raisonné de formation de cellesci informe la décision collective. Une telle thèse a suscité beaucoup de débats, que nous ne pouvons résumer ici. Ils se sont surtout focalisés sur la possibilité, la désirabilité et les conditions d'une délibération inclusive. Cette focalisation semble cependant avoir contribué à négliger le fait que prendre une décision ne peut se réduire à délibérer (ou même à négocier). Car après avoir échangé des arguments et confronté des positions, il faut aussi se résoudre en faveur d'une option qui s'imposera à tous: l'accord unanime étant improbable, il faut adopter une décision politique en passant à un vote qui tranche. Cette décision produit alors des effets contraignants sur ses destinataires, qui n'ont pas nécessairement eu part à la délibération et au vote. Prendre une décision politique, c'est en effet exercer un pouvoir de commander sur des volontés que l'on oblige (Bernardi 2013, I II), un pouvoir de gouverner. Si on prend au sérieux ce moment résolutoire du vote et ses effets contraignants, on est alors confronté à deux types de questions normatives essentielles:

a. Quelle règle de décision doit-on adopter lorsque l'on vote? La règle le plus souvent invoquée est celle de la majorité. Mais comment la justifier? Quelles en sont les limites? Ces questions ont été traitées récemment à nouveaux frais, notamment par des auteurs qui se sont attachés à penser le lien entre le processus délibératif et la règle de la majorité ${ }^{10}$.

b. Qui doit posséder le pouvoir de prendre les décisions politiques au nom du dèmos, le pouvoir de gouverner? Comment désigner et contrôler ceux qui auront ce pouvoir?

8. Sur les théories délibératives de la démocratie, voir Elster, I998; Bohman \& Regh I999; Leydet, 2002; Girard \& Le Goff, 20I0; Sintomer \& Julien Talpin, 20I I.

9. Dans un des textes fondateurs des théories délibératives de la démocratie, J. Cohen définit ainsi la démocratie:: «Une société est démocratique lorsque ses affaires sont gouvernées par la délibération publique de ses membres» (Cohen, I989, I7, notre trad.).

10. Sur la justification de la règle de majorité, voir notamment Manin, I985; Risse, 2004; Mansbridge et alii, 20I0; Girard, 20I4. 
C'est à ce second type de question que l'élection apporte une réponse, réponse à laquelle je m'intéresse ici. Une réflexion sur la légitimité démocratique ne peut en effet se focaliser exclusivement sur les conditions d'un rapport dialogique entre citoyens au sein d'un espace public ou d'une instance de délibération. Elle doit aussi penser la forme que devrait prendre le processus par lequel des agents peuvent être habilités à décider pour l'ensemble des citoyens, à les gouverner, ainsi que le rapport qui doit s'instaurer entre ceux-ci et ceux-là. Il s'agit donc d'élucider le rapport représentatif dans l'ordre de la décision - un ordre qui n'est pas réductible à celui de la délibération - afin de déterminer qui peut légitimement gouverner et dans quelles limites.

\subsection{Pourquoi la justification de l'élection demeure une question philosophique importante}

D'aucuns pourraient cependant estimer qu'une telle interrogation sur «qui doit avoir le pouvoir de prendre les décisions politiques?» et "comment désigner ces décideurs? » ne mérite guère que la réflexion philosophique s'y attarde. Soit parce que la réponse serait triviale (I); soit parce qu'elle serait secondaire, voire obsolète (2). Examinons ces deux arguments.

I) Si l'on se réfère à la définition classique du "gouvernement du peuple par le peuple ", il pourrait sembler évident qu'en démocratie, c'est le peuple - l'ensemble des citoyens - qui, en dernier ressort, devrait décider souverainement, ce pouvoir incluant celui de le déléguer, au moins en partie. L'élection serait à considérer comme la modalité de désignation qui, par excellence, exprime la souveraineté du peuple.

Pourtant, une telle position est loin d'être triviale. D'abord, parce que la question de ce qui constitue le peuple, le dèmos, est sujette à controverse et ne cesse de se compliquer dans les sociétés complexes (Goodin, 2007; Saunders, 20II): doit-on par exemple considérer que tous ceux, et seulement ceux, qui sont affectés par une décision devraient constituer le dèmos? Ou l'inclusion dans le dèmos suppose-t-elle l'appartenance à une communauté de citoyens permanente et bien circonscrite? Ne devrait-on pas apposer des limites au droit de suffrage? (Lopez-Guerra, 20I4). De plus, la question de l'habilitation à décider pour le dèmos et à la place de celui-ci, donc de l'habilitation à gouverner, n'a pas une réponse aussi triviale, comme en attestent précisément les nombreuses controverses sur l'aptitude des élections à conférer une telle habilitation, notamment dans les cas où ceux qui sont affectés par une décision sont dans l'impossibilité d'y prendre part directement ou indirectement, comme c'est le cas pour les générations futures ${ }^{11}$.

11. Sur la représentation des générations futures, voir Gonzalez-Ricoy \& Gosseries, 2017. 
2) On pourrait, par ailleurs, considérer que se demander «qui doit avoir le pouvoir de prendre les décisions politiques » est devenu aujourd'hui secondaire et, pour ainsi dire, obsolète. En effet, les évolutions politiques au cours des dernières décennies seraient caractérisées par une complexification et une pluralisation des modalités de la décision publique et des acteurs qui y participent. Il apparaîtrait ainsi que désormais la décision publique serait à concevoir de façon processuelle et que le pouvoir de décision ne pourrait donc se concentrer dans les mains d'un seul agent mais serait distribué entre des agents de statuts différents (publics, privés, associatifs, etc.), interagissant en réseaux, intervenant et rétroagissant à des moments différents de processus de décision demeurant ouverts. Selon ces approches contemporaines de la "gouvernance multi-niveaux", nous devrions renoncer à la thèse simpliste selon laquelle l'action publique serait entièrement déterminée, "gouvernée» par les parlements et les gouvernements (éventuellement contrôlés par des cours) détenteurs des attributs de la souveraineté. Les rôles et les pouvoirs de ceux-ci seraient en train de se réduire, laissant la place à d'autres acteurs et d'autres instances ne procédant pas de l'élection: autorités administratives indépendantes, juridictions, organisations internationales, forums associant acteurs privés et publics, etc. ${ }^{12}$ L'absence de légitimation de ces instances par l'élection ne les rendrait pas pour autant non démocratiques. Car la légitimité démocratique devrait, elle aussi, être pensée de manière pluralisée (Rosanvallon, 2008): la volonté du peuple pouvant être représentée et se manifester de manières diverses, dans des procédures «dépersonnalisées » - les agents habilités à intervenir dans la décision pouvant être désignés par des voies qui le sont tout autant. La question des modalités de désignation des acteurs qualifiés pour intervenir dans la décision deviendrait dès lors secondaire. L'essentiel serait plutôt que les interventions des différents agents, officiels ou non, puissent être contrôlées au regard des attentes de légitimité que l'on a envers eux. Ainsi, l'impartialité attendue des autorités publiques ne pourrait être assurée par l'élection, mais plutôt par la surveillance exercée par les acteurs de la société civile, par des juridictions ou tout simplement par la distribution du pouvoir entre des intervenants qui se contrôlent mutuellement.

Je ne puis ici discuter de manière approfondie ces thèses sur la fragmentation du pouvoir politique et sur la dissolution du rôle central classiquement dévolu aux institutions gouvernementales et parlementaires. Je voudrais néanmoins indiquer trois raisons qui amènent à penser qu'une société démocratique ne peut renoncer à de telles institutions.

a) Il y a manifestement des enjeux sur lesquels le pouvoir de décision politique et l'action des institutions gouvernementales des États demeurent essentiels et où ils pourraient difficilement être remplacés par une gouvernance en réseau (Rosanvallon 20 I 5, I8I). On pense à ce qui touche, entre

12. Pour une présentation critique des approches, voir Papadopoulos, 20 I० \& 20 I 2. 
autres, aux fonctions régaliennes (police, justice, défense, etc.) et aussi surtout aux pouvoirs qui permettent de définir des priorités et d'assurer une continuité et une cohérence dans les politiques publiques. Ces pouvoirs se manifestent notamment au moment où un gouvernement établit et présente son budget et, ce faisant, hiérarchise les objectifs politiques qu'il se donne, même si cela se produit dans un contexte économique très contraignant. Il semble donc que nos sociétés démocratiques de masse ont besoin d'instances gouvernementales centrales. Je donne ici à cette notion un sens très large qui ne la limite pas au pouvoir exécutif tel qu'on le définit classiquement (sur ce sujet, voir Rosanvallon, 20I 5) mais inclut les parlements et peut couvrir une grande diversité de formes d'organisation politique. Il s'agit de ces instances permanentes généralistes qui disposent formellement du pouvoir d'orienter l'action publique dans tous les domaines et d'en assurer la continuité et la visibilité pour et au nom de l'ensemble du dèmos.

b) De telles instances de gouvernement ne répondent pas seulement au besoin d'une action publique cohérente et efficace. Leur importance pourrait aussi se justifier par la nécessité de figures personnalisées du pouvoir politique. Les procédures de gouvernance multi-niveaux se prêtent mal à l'identification d'agents responsables des décisions: celles-ci sont en effet le fruit d'interactions continues entre un nombre important d'intervenants de statuts souvent très différents. À l'inverse, les institutions gouvernementales et parlementaires reposent sur l'imputation publique d'un pouvoir et d'une responsabilité politique importante à des représentants et des gouvernants qui ont des comptes à rendre et dont l'action (ou l'inaction) peut être contestée et sanctionnée par les citoyens. Même si cette imputabilité n'est pas dépourvue d'effets pervers, un certain degré de personnalisation du pouvoir rend plus commode l'exercice du contrôle sur celui-ci ${ }^{13}$.

c) Enfin, si la décision et l'action publiques passent, de plus en plus, par des procédures de gouvernance en réseau "multi-niveaux", le caractère démocratique de celles-ci est pour le moins contesté (Papadopoulos, 20Io et 20I2). La possibilité effective pour le citoyen d'agir et d'influer sur la décision y est souvent très faible et/ou inégalement distribuée. Ces procédures échappent pour la plupart à son emprise. Certaines d'entre elles associent bien entendu des acteurs ou organisations de la société civile, censés alors représenter les intérêts et les points de vue des citoyens. Mais la sélection des acteurs ou organisations impliqués est souvent opaque et discutable; les pouvoirs et les ressources dont ils disposent sont inégalement répartis et leur représentativité n'est pas toujours bien assurée. À l'inverse, le fonctionnement et les pratiques des gouvernements et des parlements sont davantage

13. Parmi ces effets pervers, on mentionnera la «peoplisation» de la vie publique qui abolit la distinction entre le privé et le public, ou encore la tendance populiste à occulter la nécessité de médiations institutionnelles pour la représentation démocratique. 
exposés au regard des citoyens, plus lisibles également, et donc susceptibles d'un contrôle démocratique plus commode.

Les raisons que je viens de mentionner donnent à penser que nos sociétés complexes de masse ont besoin d'instances gouvernementales et que celles-ci doivent y occuper une place centrale dans le système politique. L'existence même d'une communauté politique démocratique ainsi que la continuité et l'imputabilité de l'action publique semblent requérir ces institutions permanentes, un lieu où toutes les questions et les revendications puissent être portées, débattues, même si elles n'y sont pas formellement toutes décidées, au nom de tous. La question des modalités légitimes de désignation de celles et ceux qui siègent dans ces lieux n'est donc pas anecdotique. Mais si la réponse apportée, depuis près de deux siècles, fut celle de l'élection des gouvernants, on peut néanmoins légitimement se demander si, au vu des critiques qui lui sont adressées, elle demeure appropriée. Une société démocratique de masse pourrait-elle se passer complètement d'élections pour désigner ses gouvernants?

Mais quelle serait alors la solution de rechange?

\subsection{L'option du tirage au sort}

On a pratiqué par le passé - et on peut aussi imaginer de manière prospective - d'autres modalités de désignation des gouvernants: cooptation, concours, succession héréditaire, acclamation, etc. Mais la modalité démocratique qui semble aujourd'hui la plus invoquée par ceux qui mettent en question l'élection est la sélection aléatoire au sein du corps des citoyens. Le tirage au sort est en effet présenté par d'aucuns comme un remède aux errements de la démocratie électorale. Il est certes très rare qu'on le considère comme un substitut pur et simple à l'élection ${ }^{14}$. En revanche, on a vu fleurir, au cours des dernières années, plusieurs propositions visant à mettre en place, dans nos systèmes politiques, des parlements de citoyens sélectionnés de manière aléatoire qui disposeraient de réels pouvoirs en matière politique, venant ainsi compléter, contrebalancer ou remplacer le pouvoir des instances élues dans toute l'étendue de leurs compétences ${ }^{15}$. Il ne s'agit donc pas simplement de propositions visant à utiliser le tirage au sort pour constituer ponctuellement des jurys, des conférences de citoyens ou des échantillons de sondages délibératifs à vocation informative ou consultative sur un enjeu politique circonscrit ${ }^{16}$. On voit en effet surgir des projets plus ambitieux qui viseraient à associer des citoyens « ordinaires", choisis au hasard, dans une assemblée de type parlementaire dotée d'un pouvoir de décision étendu — ou, à tout le moins, un droit de veto — en matière de législation

14. Voir toutefois une initiative référendaire en Suisse: http://www.genomi.ch/ (consulté le 6 novembre 20I5).

15. Voir Van Reybrouck, 20I2; Sintomer, 2012.

16. Sur ce genre de dispositif, voir notamment Pourtois, 20I3. 
et/ou de contrôle de l'exécutif. Il s'agirait de transférer, en tout ou en partie, le pouvoir aujourd'hui détenu par des élus à des parlementaires tirés au sort.

Ceux qui promeuvent un tel projet invoquent plusieurs arguments ${ }^{17}$ : I) le tirage au sort conférerait à l'assemblée une représentativité sociologique: sa composition sociale serait vraisemblablement plus proche de celle du dèmos que dans le cas des assemblées aujourd'hui élues; 2) la sélection aléatoire permet en outre d'égaliser les chances pour les citoyens de prendre une part active à la vie publique en exerçant un mandat et ce, quelle que soit leur origine sociale, alors que les mandats électifs semblent aujourd'hui quasiment réservés à des citoyens issus des classes supérieures ou moyennes; 3 ) la diversité sociale que le tirage au sort engendre au sein de l'assemblée contribuerait aussi à accroître la diversité des perspectives, des expériences et des savoirs présents dans l'assemblée, et par là, à améliorer la qualité épistémique de ses délibérations et décisions; 4) le tirage au sort permettrait d'éviter les effets pervers de la dynamique de professionnalisation présente dans nos systèmes représentatifs: en mettant des "citoyens ordinaires " au cœur de la décision, elle éviterait la mainmise d'une classe politique avec ses intérêts propres sur le système politique; 5) le recours au tirage au sort, finalement, neutraliserait les logiques partisanes, qui pour d'aucuns pervertissent la délibération politique ${ }^{18}$.

Nous avons donc certainement de bonnes raisons de prendre au sérieux des propositions qui promeuvent une assemblée parlementaire issue d'un tirage au sort parmi les citoyens. Mon intention n'est cependant pas de présenter et de discuter ici, de manière systématique et dans le détail, les avantages d'une telle option et les objections qu'on peut lui adresser.

Plus modestement, je me propose plutôt d'essayer d'identifier quelquesunes des vertus spécifiques de l'élection des parlements au suffrage universel, vertus qui sont ou seraient, selon toute vraisemblance, absentes d'autres modes démocratiques de désignation des gouvernants, du tirage au sort en tout cas. Dans cet exercice, la proposition d'une assemblée parlementaire tirée au sort ne me servira que d'outil heuristique destiné à identifier en creux les vertus démocratiques que possède l'élection et qui ne pourraient être assumées par une telle assemblée. Mon but n'est donc pas de confronter les bienfaits et les faiblesses des deux systèmes de désignation ni d'examiner leurs combinaisons possibles et souhaitables, mais d'identifier les potentialités démocratiques incluses dans la dynamique électorale et spécifiques à celle-ci. Si j'use ici du vocabulaire aristotélicien en évoquant les «vertus» ou les "potentialités", c'est tout simplement parce que les qualités propres à l'élection des gouvernants au suffrage universel peuvent ou non se manifester en fonction des modalités d'organisation de celle-ci et des conditions

17. Pour une présentation systématique des arguments, voir Sintomer, 20 I I, I 92 et sq.

18. Notons toutefois que certains considèrent que la logique partisane peut contribuer, plutôt que nuire, à la qualité de la délibération démocratique (White \& Ypi, 20I I). 
sociales du contexte. L'identification de ces conditions est cependant une tâche trop importante pour être entreprise ici. Les arguments développés suggèreront cependant que la présence de partis politiques est essentielle au déploiement des vertus de l'élection. Mais notre propos ne sera pas de démontrer cette thèse. La seconde partie de cet article présentera en effet avant tout un plaidoyer visant à montrer pourquoi les théories normatives de la démocratie devraient se préoccuper davantage des élections.

\section{Les vertus démocratiques de l'élection}

Afin d'identifier les vertus démocratiques de l'élection, je vais comparer celle-ci au tirage au sort au regard de leurs aptitudes respectives à satisfaire quatre critères relatifs à la légitimité démocratique d'une procédure représentative de décision politique. Il existe de fait différentes conceptions de la légitimité démocratique ${ }^{19}$. Elles n'accordent pas la même signification et la même valeur aux critères que je propose. Je n'opterai cependant pas ici pour l'une ou l'autre de ces conceptions, préférant laisser le débat ouvert sur ce point - y compris sur le point de savoir s'il ne faudrait pas ajouter d'autres critères. Il me semble toutefois que l'on pourra aisément se rallier à ces critères, au moins à certains d'entre eux ${ }^{20}$.

Les quatre critères sont:

I. Le consentement des citoyens à être gouvernés;

2. L'inclusion égalitaire des citoyens dans le processus de décision;

3. Le contrôle et la contestation des décisions et des décideurs par les citoyens;

4. Les qualités épistémiques du processus de décision.

\subsection{Le consentement à être gouverné}

En démocratie, ceux qui font les lois et disposent d'un pouvoir de commander, de gouverner les citoyens, ne peuvent en disposer que moyennant le consentement de ceux-ci. L'élection des gouvernants est souvent vue comme l'expression paradigmatique, voire le garant exclusif, de ce consentement. En élisant une assemblée (un député), les citoyens autorisent, habilitent celle-ci (celui-ci) à prendre, en leur nom, des décisions contraignantes aux-

19. Voir, par ex., Weale, 2007.

20. Notre ambition n'étant pas de présenter ici une théorie générale et complète de la démocratie, il ne nous paraît pas utile de justifier le choix de ces quatre critères autrement que par le fait qu'ils sont souvent invoqués dans les débats sur le sens et la valeur de la démocratie. Nous sommes bien conscients que l'un ou l'autre de ces critères pourrait être contesté ou que l'on pourrait en proposer d'autres formulations ou encore ajouter d'autres critères. Ainsi l'affirmation selon laquelle les procédures démocratiques devraient avoir des vertus épistémiques est mise en cause par d'aucuns (Saffon \& Urbinati, 20I3). Il suffit cependant à notre propos que le lecteur puisse reconnaître dans ces critères - au moins l'un d'entre eux — des positions raisonnables sur ce que suppose une procédure démocratique légitime. 
quelles ces citoyens électeurs devraient se conformer. Telle est la justification majeure que produisaient les promoteurs du gouvernement représentatif en faveur de l'élection et contre le recours au tirage au sort (Manin, I995, ro8 ss.). La désignation par le sort serait en effet, comme telle, dépourvue de toute force d'autorisation. C'est la raison pour laquelle certains auteurs (Parkinson 2006, 4I ss.) excluent de donner un pouvoir de décision à une instance qui, comme un mini-public désigné par le sort, n'a pas été autorisée par une élection.

Cet argument classique semble cependant avoir une portée limitée.

En effet, le pouvoir conféré à une institution publique ou à un agent public ne repose pas toujours et ne doit pas nécessairement reposer sur un consentement direct de ceux qui y sont soumis. L'autorisation peut être indirecte. Dans de nombreux pays, les juges constitutionnels ou les responsables des banques centrales, qui ont pourtant des pouvoirs importants, ne sont pas élus. On peut néanmoins admettre qu'ils sont démocratiquement autorisés à exercer les pouvoirs que leur donne la Constitution en vertu du fait que les citoyens adhèrent à celle-ci, même si ce consentement est implicite. On peut imaginer qu'il en irait de même avec une assemblée tirée au sort, les citoyens s'accordant sur le fait que seule cette assemblée serait autorisée à faire les lois. L'habilitation serait, comme dans les autres cas mentionnés, indirecte.

Mais, de fait, elle l'est également dans le cas de l'élection. Au sens strict, les députés élus n'ont obtenu un consentement direct à pouvoir gouverner que des citoyens qui les ont élus dans leur circonscription. Ce qui fait finalement peu de monde par rapport à l'ensemble de l'électorat. En revanche, on peut dire que si une assemblée est légitime et peut donc prendre des décisions qui s'imposent à tous, c'est parce que ses membres ont été désignés au terme d'une procédure électorale que les citoyens reconnaissent comme légitime. Mais ils pourraient, tout compte fait, adopter une autre procédure, comme le tirage au sort, par exemple. C'est donc le consentement à l'égard d'une procédure de sélection plutôt qu'à l'égard d'une candidature qui habilite des mandataires à décider collectivement pour le dèmos.

Le principe du consentement à être gouverné ne semblerait donc pas absolument déterminant pour le maintien de l'élection des gouvernants. Cependant, on pourrait lui trouver une vertu particulière. L'élection suppose le vote des citoyens et donc un choix opéré par chacun d'entre eux, y compris le choix éventuel de ne pas voter. Même si, dans une démocratie de masse, l'impact du choix de chacun sur la désignation des gouvernants et sur les politiques qu'ils mènent est extrêmement faible, ce choix individuel signifie néanmoins une participation à la coresponsabilité pour les actions posées, ou pas, par le gouvernement ou l'opposition. Je peux être en désaccord avec une décision prise par mon gouvernement, il n'en reste pas moins que je partage une part de responsabilité dans le fait qu'il occupe le pouvoir, surtout si je lui ai donné mon suffrage. L'élection contribuerait ainsi à la 
création et à la production permanente d'une communauté de responsabilités. Cette imputation peut se révéler utile, par exemple, dans le contexte des relations internationales ou dans les cas de fautes graves posées par des gouvernements. On imputera plus facilement à un peuple, à une communauté de citoyens, une responsabilité pour les actes commis par son gouvernement si celui-ci est issu de l'élection plutôt que, par exemple, le fruit d'un coup d'État ou du hasard de la génétique. Par analogie, on pourrait considérer que la désignation des gouvernants par le tirage au sort, puisqu'elle ne permet pas au citoyen de poser un choix, d'influer sur cette désignation, ne les inscrit pas dans une communauté de responsabilité à l'égard des politiques.

\subsection{L'inclusion égalitaire des citopens dans le processus de décision}

Une procédure de décision est démocratique si elle est inclusive: si elle assure à tous les citoyens, quelle que soit leur origine sociale, une possibilité égale de prendre part, directement ou indirectement, à la formation de la décision politique.

Tocqueville soulignait ${ }^{21}$ que l'égalité politique est au cœur de la démocratie et implique le suffrage universel. Et de fait, la mise en œuvre progressive de celui-ci à l'occasion de l'élection des gouvernants fut le principal vecteur de la démocratisation de nos sociétés aux $\mathrm{XIX}^{\mathrm{e}}$ et $\mathrm{XX}^{\mathrm{e}}$ siècles. Depuis lors, on a cependant pu se rendre compte que l'élection au suffrage universel n'est pas vraiment inclusive. Trois raisons sont souvent avancées: a) de facto, la participation électorale est socialement biaisée: les citoyens plus scolarisés et issus de milieux plus favorisés votent davantage que ceux qui sont moins scolarisés et issus de milieux moins favorisés; b) le même biais social affecte, de manière encore plus marquée, l'accès aux mandats politiques: la grande majorité des élus ont un niveau de revenu et un niveau d'études supérieurs à la moyenne des citoyens; ils ont souvent fréquenté les mêmes écoles, les mêmes cercles et sont parfois issus des mêmes familles; c) enfin, la dynamique électorale conduit à une professionnalisation de la politique, à la constitution d'une "classe politique » dotée d'un certain pouvoir social et dont les intérêts et le mode de vie se distinguent de ceux du reste de la population et parfois s'y opposent. Ces phénomènes sont bien connus, même si leur forme et leur ampleur varient selon les systèmes politiques ${ }^{22}$.

Le recours au tirage au sort pour la formation d'une assemblée est parfois invoqué comme remède à ces biais. Il permettrait d'égaliser les chances de participation à la vie publique et à la décision pour tous les

21. "Il est impossible de comprendre que l'égalité ne finisse pas par pénétrer dans le monde politique comme ailleurs. On ne saurait concevoir les hommes éternellement inégaux entre eux sur un seul point, égaux sur les autres; ils arriveront donc, dans un temps donné, à l'être sur tous» (Tocqueville I 835, I, I ${ }^{\text {re }}$ partie, chap. III).

22. Voir notamment les travaux de Meyer, 2010; Norris, 2002. 
citoyens, quelle que soit leur origine sociale, et d'éviter les effets pervers induits par la professionnalisation de la politique et son accaparement par les partis. L'ouvrier, la ménagère ou le sans-emploi auraient autant de chances que l'universitaire de siéger dans une assemblée. De plus, la diversité sociale ainsi induite et le mode de recrutement aléatoire devraient réduire les risques de formation d'une classe politique coupée des réalités et des expériences vécues par le reste de la population: le parlement pourrait devenir une assemblée de "citoyens ordinaires".

Certes, on doit relever que, dans les quelques cas récents où l'on a recouru au tirage au sort pour composer un jury ou une assemblée de citoyens, on n'a pas réussi à réduire complètement les biais sociaux mentionnés. Il s'avère en effet très difficile de composer un échantillon pleinement représentatif en raison d'un biais d'auto-(dé)sélection (Blondiaux, 2008): les moins bien lotis ou scolarisés renoncent plus souvent que les autres à participer alors même que l'occasion leur est offerte. La participation aux travaux d'une assemblée ou d'un jury suppose en effet l'acceptation et l'adhésion des participants sélectionnés. Or on constate que les plus défavorisés expriment souvent leur refus et que lorsqu'ils acceptent bon gré mal gré de prendre part à ce genre d'expérience, ils ont tendance à s'y engager moins activement, estimant qu'ils ne possèdent pas les compétences requises $^{23}$. On peut faire l'hypothèse que cette difficulté serait accrue, certainement pas réduite, dans le cas de l'implication, plus exigeante et chronophage, requise par le fait de siéger dans une assemblée parlementaire tirée au sort. À partir des quelques expériences ponctuelles d'assemblées ou de jurys de citoyens, on observe aussi que ceux qui acceptent d'y participer sont celles et ceux qui sont intéressés à le faire et en ont la possibilité réelle ${ }^{24}$. Cet intérêt et cette possibilité sont variables selon les milieux sociaux. Reconnaissons toutefois qu'il est hautement probable que la composition sociale d'une assemblée tirée au sort serait plus diversifiée et plus proche de celle de la société que la composition actuelle des assemblées élues. Le remplacement de l'élection par le tirage au sort, a fortiori si ce dernier est assorti de mécanismes d'échantillonnage, permettrait donc de corriger assez rapidement et significativement, bien qu'imparfaitement, l'inégalité dans l'accès à la représentation.

On doit toutefois se demander si le jeu en vaut la chandelle. Il est en effet à craindre qu'un tel remplacement (a) ne soit pas nécessaire (b) ne soit pas sans risque.

a) Tout d'abord, il n'est pas inimaginable que l'on puisse, dans le cadre d'une démocratie élective, mettre en place des règles et des dispositifs qui réduiraient le biais social dans la participation électorale et l'accès à la représentation. Des efforts ont déjà été faits en ce sens pour ce qui concerne le

23. Sur ce phénomène d'" auto-désahabilitation ", voir Gaxie, 2007.

24. Des retraités ou des étudiants, par exemple, qui ont un emploi du temps plus flexible. 
genre ou la diversité ethnique. Il y a quelques décennies, les mandats dans les assemblées parlementaires et les gouvernements étaient presqu'exclusivement occupés par des mâles blancs d'âge mûr. Depuis ce temps, sous l'effet de mouvement sociaux, de politiques incitatives et parfois de réglementations, la présence des femmes et des minorités ethniques ou issues de l'immigration n'a cessé de s'accroître. La plupart des pays n'ont certes pas encore réalisé la parité de genre dans les mandats politiques, mais ils en sont plus près qu'il y a vingt ans. Il n'est donc pas interdit de penser que l'on pourrait aussi voir - et en fait, on le voit déjà - surgir des revendications et des pratiques visant à réduire l'impact des différences d'origine sociale sur la participation politique et l'accès aux mandats publics. La mesure la plus radicale en ce sens serait évidemment de remplacer l'élection par le tirage au sort. Mais ce n'est certainement pas le seul moyen d'atteindre l'objectif recherché. Et surtout, cette solution radicale nous priverait d'un vecteur important de participation politique.

b) On doit en effet aussi se demander ce qu'il adviendrait si l'on supprimait ou réduisait significativement la possibilité pour les citoyens d'élire leurs gouvernants. De fait, cela reviendrait à priver ces citoyens d'une occasion de participer à la vie publique et d'influer sur la décision politique qui est accessible à tous sans autre condition que d'être citoyen. Cela les priverait eo ipso d'une expérience sociale partagée, vécue simultanément par eux de manière réitérée et régulière au cours de leur existence: l'expérience de la campagne électorale et du scrutin qui la clôture. Le tirage au sort et la participation à une assemblée désignée par cette voie ne peuvent remplacer, dans son amplitude sociale, la participation qui peut être expérimentée à l'occasion d'un scrutin électoral.

Le tirage au sort offre bien sûr une chance relativement égale pour chaque citoyen de participer à la décision publique et de vivre ainsi une expérience politique riche et édifiante. Cependant, dans une société de masse, seule une très faible proportion de citoyens pourrait effectivement accéder à un mandat de représentant à l'assemblée parlementaire, que celleci soit élue ou tirée au sort. Si on postule la nécessité d'une assemblée stable, permanente et susceptible de débattre, le nombre de citoyens ayant l'occasion d'y siéger ne sera pas très significatif et ce, quelle que soit l'égalisation des chances d'y siéger. C'est d'ailleurs la différence fondamentale avec le tirage au sort tel qu'il était pratiqué à Athènes, où le nombre limité de citoyens et la rotation fréquente des charges permettaient à la fois d'égaliser les chances et d'associer un très grand nombre de citoyens à l'exercice des charges publiques (lesquelles n'étaient toutefois pas législatives) ${ }^{25}$. Même si l'on adoptait, aujourd'hui, la règle selon laquelle un citoyen ne peut siéger que pour une seule mandature dans une assemblée parlementaire tirée au

25. Il semblerait qu'un citoyen avait plus d'une chance sur deux d'exercer au moins une fois une telle charge. 
sort, la proportion des citoyens qui auraient l'occasion de le faire resterait très faible et ne pourrait être sensiblement plus élevée que dans le cas d'une assemblée élue.

En revanche, l'acte de voter lors d'une élection est accessible à tous les citoyens sans condition et les associe à une décision politique importante: celle du choix des gouvernants. Le suffrage universel ne donne pas à chacun une probabilité égale, mais une possibilité effective relativement égale de participer. Certes, la participation électorale est socialement biaisée et semble tendanciellement en recul dans beaucoup de pays ${ }^{26}$. Mais il n'en reste pas moins que voter aux élections demeure la forme de participation politique qui est à la portée de tous, qui est la plus largement utilisée par les citoyens et qui semble la moins affectée par l'origine sociale et le niveau d'études si on la compare à d'autres formes de participation politique: pétitionner, manifester, militer dans un parti ou une association, etc. (Meyer 20I0). Voter, c'est «exercer un pouvoir de manière individualisée et inconditionnée» (Pettit, 20I3). Et le suffrage universel distribue ce pouvoir de manière égale entre tous, au moins formellement, et ce, quels que soient les mérites, les compétences, les besoins ou l'origine sociale. Supprimer cette forme de participation simple et largement distribuée, ou même réduire significativement sa portée en rabotant le pouvoir des élus et donc l'enjeu de l'élection ne serait certainement pas sans effets sur les citoyens et sur la communauté politique.

Tout d'abord, cela priverait les citoyens d'une possibilité d'exercer, s'ils le souhaitent, une influence sur les affaires publiques, distribuée de manière égale entre eux, et qui n'est formellement dépendante d'aucune autre condition que celle d'avoir le statut de citoyen adulte, à l'exclusion de toute autre condition de formation, de compétence ou de mérite présumés ou encore d'appartenance à un parti, une association, etc. Si le tirage au sort lève lui aussi toutes ces conditions, il ne permet cependant pas au plus grand nombre de citoyens d'exercer de fait le pouvoir d'influence que confère le droit de vote.

De plus, l'acte de voter ne donne pas seulement un pouvoir au citoyen. Il contribue aussi à sa socialisation et à la constitution d'une véritable communauté politique. Le suffrage universel accomplit ce que Pierre Rosanvallon a appelé "le sacre de l'égalité». En assurant une égalité d'influence, il confère aussi une égalité de statut: il contribue à ce que les citoyens se reconnaissent mutuellement comme des égaux (ce dont attestent par la négative les situations où certains sont privés de ce $\left.\operatorname{droit}^{27}\right)$. Contrairement à ce qu'a parfois avancé la critique marxiste de la démocratie représentative, l'égalité de pouvoir et de statut induite par le suffrage universel n'est donc pas purement abstraite. En effet, voter ne se réduit pas à exprimer une préférence ou

26. Voir Mair, 20I3.

27. Voir Honneth, I999. 
une opinion que l'élection viserait à agréger à celles des autres citoyens en équilibrant le pouvoir de chacun. Voter aux élections est une pratique sociale codifiée et ritualisée qui met en jeu des rapports sociaux, entre les citoyensélecteurs et entre ceux-ci et les acteurs politiques (Cf. Deloye \& Ihl, 2008; Ihl, 20I0; Faucher \& Hay, 20I5). L'ensemble des pratiques électorales et des pratiques qui l'entourent ${ }^{28}$ peuvent ainsi avoir des effets positifs sur la socialisation politique des citoyens et leur propension à se reconnaître comme égaux.

Bien entendu, on pourrait objecter que la suppression des élections n'impliquerait pas la disparition du vote des citoyens: ceux-ci pourraient continuer à s'exprimer lors de référendums ou de consultations populaires, que la disparition de l'élection rendrait peut-être même plus fréquents. Il y a toutefois une différence importante entre le vote électif et le vote référendaire: nous aurons l'occasion de le montrer plus loin.

\subsection{Le contrôle des décideurs et la contestabilité des décisions}

Dans une société démocratique, tous les citoyens doivent avoir un droit de contester, et, ce faisant, de contrôler, les décisions politiques; et ceux qui les prennent doivent rendre des comptes sur ces décisions. L'élection assure cette redevabilité (accountability) ${ }^{29}$ à l'égard de l'ensemble des citoyens et donne à chacun d'entre eux un pouvoir de contrôle et de contestation.

La redevabilité des gouvernants et des représentants est mimimalement assurée par le fait que leurs mandats sont attribués pour une durée déterminée au terme de laquelle l'électeur peut ne pas souhaiter renouveler le mandat des sortants.

La contestabilité $e^{30}$ suppose la possibilité pour les citoyens de poser un jugement autonome sur les affaires publiques et les choix opérés par les gouvernants; elle suppose aussi que ces mêmes citoyens disposent de moyens de contrôle ou d'influence sur ces choix. Ils doivent demeurer libres de mettre en question et d'infléchir une décision qui ne prendrait pas en considération leurs préférences, intérêts ou arguments, et ce, même si la décision est prise par une autorité publique dûment désignée et habilitée à la prendre.

On pourrait avancer que la redevabilité et le pouvoir de contrôle que l'élection confère au citoyen demeurent très formels et relativement faibles puisque ce dernier ne peut s'exprimer qu'une fois tous les quatre ou cinq ans en déposant son bulletin dans l'urne. Ce qui laisserait, entre deux échéances électorales, une très - trop - grande liberté pour les élus de gouverner à leur guise, sans considérer l'intérêt public ou le souhait des citoyens. Il est

28. Voir par ex. les travaux de P. Cossart sur les meetings comme lieux de participation et de socialisation politiques.

29. La littérature sur la notion d'accountability est importante. On pourra se référer notamment à Przeworski et al., I999, ou encore à Bovens, 2007.

30. Voir Pettit, 2013. 
vrai qu'il est dans la nature même de la démocratie représentative de déléguer un pouvoir aux représentants, et donc de leur donner, de droit et de fait, une indépendance par rapport à ceux qu'ils représentent ${ }^{31}$. Cependant l'élection instaure également un lien, une interaction permanente entre les représentants et les représentés. Comme le souligne Bernard Manin, le principe de la réitération régulière de l'élection permet aux citoyens d'influer $d e$ manière continue sur la décision publique en vertu du mécanisme suivant:

des représentants soumis à réélection sont incités à anticiper le jugement rétrospectif des électeurs sur la politique qu'ils mènent. La perspective d'un renvoi possible exerce par anticipation un effet sur la conduite des gouvernants à tous les moments de leur mandat (Manin, I995, 228).

Même si tous les sortants ne sollicitent pas un renouvellement de leur mandat, le mécanisme mis en avant par B. Manin s'applique certainement aux partis politiques au pouvoir, dont l'objectif premier est d'y demeurer. L'anticipation du jugement rétrospectif des électeurs sur les politiques menées devrait inciter les gouvernants ${ }^{32}$ à agir de deux manières: a) en adoptant des politiques dont ils peuvent escompter qu'elles recevront un jugement rétrospectif favorable de l'électeur, à la prochaine échéance électorale (et ce même si elles sont contestées au moment où elles sont décidées); b) en s'efforçant de convaincre les citoyens du bien-fondé d'une politique «dont ils n'ont pas l'idée ou ne veulent pas » mais qui semble pourtant essentielle pour le bien public.

Évidemment, le mécanisme décrit par B. Manin est un modèle idéaltypique dont les pratiques électorales en vigueur peuvent parfois s'écarter fortement et dont la réalisation suppose que soient satisfaites une série de conditions relatives aux modalités du scrutin et aux conditions sociales dans lequel il se déroule. Ce mécanisme suppose, par exemple, que les modalités de scrutin ne confèrent pas un avantage excessif $\mathrm{au}(\mathrm{x})$ parti(s) au pouvoir qui rendrait l'alternance difficile. Cependant le mécanisme d'anticipation du jugement rétrospectif nous montre que la dynamique électorale génère une interaction entre gouvernants et gouvernés qui induit fortement les premiers à tenir compte des jugements attendus des seconds et à justifier leur action à leur égard; ce qui donne, en retour, aux gouvernés une possibilité d'influer sur les gouvernants. Cette influence induite par la dynamique électorale ne se manifeste du reste pas seulement par la voie des urnes au moment de l'élection. Elle s'incarne également dans l'action autonome des citoyens s'associant dans l'espace public: l'expression publique de jugements politiques suscitée par la dynamique électorale favorise en effet l'association de citoyens autour de causes ou de convictions partagées. La procédure, extrêmement

31. La question du niveau d'indépendance faisant toutefois l'objet de débats...

32. Ou en tout cas, inciter les partis politiques au pouvoir à faire pression sur leurs ministres et leurs députés à agir dans le sens indiqué. 
formalisée, de l'élection contribuerait ainsi fortement à la constitution non seulement d'interactions entre gouvernants et citoyens, mais aussi d'interactions et de coopérations informelles entre les citoyens au sein de l'espace public. Bien entendu, ces potentialités ne peuvent se déployer que si certaines conditions caractérisant le scrutin sont remplies.

En tout état de cause, il semble que le recours au tirage au sort pour la désignation de gouvernants ou de représentants ne possède pas de vertus équivalentes.

Les décisions prises par une assemblée tirée au sort ou par ses membres pourraient-elles être sujettes à une redevabilité de ceux-ci et au contrôle correspondant des citoyens? Certains auteurs, comme Parkinson (2006), répondent par la négative. Pourtant, le modèle politique athénien semble suggérer le contraire. De fait, à Athènes, les bouleutes, désignés par le sort, étaient sujets à des procédures de reddition de comptes particulièrement exigeantes, tant en cours de mandat qu'à l'issue de celui-ci. Les décisions qu'ils avaient prises pouvaient être annulées ou modifiées par l'Ecclesia, par l'assemblée des citoyens. De plus, ces mandataires pouvaient être personnellement sanctionnés, par une peine de bannissement par exemple, pour les "mauvaises» décisions qu'ils avaient prises (Hansen, 2009, 2I9 ss.). On peut bien imaginer, sans aller jusqu'au bannissement, que les décisions adoptées par une assemblée tirée au sort puissent être contestées et modifiées et que ses membres puissent être sanctionnés politiquement, par exemple au moyen d'une procédure de révocation ( recall $)^{33}$. Dans de telles conditions, qu'ils aient été sélectionnés par le sort ou élus, les magistrats devraient, dans l'exercice de leur charge, anticiper le jugement rétrospectif que les citoyens porteraient sur eux en cours de mandat, et surtout au terme de celui-ci.

Il n'est donc pas impossible de prévoir des dispositifs de contrôle et de sanction dans le cas d'une assemblée tirée au sort. On doit toutefois noter que la sanction électorale, à la différence des mécanismes de révocation, instaure un contrôle automatique régulier et exercé par l'ensemble du dèmos. Elle force en quelque sorte les citoyens à exercer périodiquement leur pouvoir de contrôle et de sanction. De plus, la compétition partisane crée une forte incitation à tenir compte du jugement de l'électeur, en dépit même de la décision de certains candidats de ne pas se représenter.

Mais la difficulté principale du contrôle des mandataires tirés au sort tient plutôt aux motifs de contestation que l'on pourrait invoquer pour le justifier. Il faut en effet s'interroger sur ce qui pourrait fonder la contestation

33. Le recall désigne la possibilité pour un électorat de mettre un terme à un mandat public avant l'échéance normalement prévue. Cette disposition est inscrite dans la Constitution de plusieurs des États aux États-Unis pour ce qui concerne le mandat des gouverneurs. Si le recall est pratiquement utilisé dans le cadre de la démocratie électorale - une désignation par élection -, il serait imaginable de l'utiliser dans le cadre du tirage au sort: désignation par tirage au sort et éventuelle révocation par scrutin. 
et la sanction éventuelle à l'égard des décisions prises par une assemblée tirée au sort ou des positions prises par ses membres. Je ne parlerai pas ici du contrôle de constitutionnalité et du respect des droits fondamentaux auquel une assemblée tirée au sort peut être soumise comme le serait un parlement élu. Dans un cas comme dans l'autre, il y a des règles du jeu et des droits fondamentaux qu'une simple majorité politique doit respecter. Je veux plutôt évoquer le fondement du contrôle politique, par les citoyens, de ceux qui sont censés les représenter et décider en leur nom. De ce point de vue, on peut observer une différence principielle entre une assemblée élue et une assemblée tirée au sort.

Dans une assemblée élue, les représentants sont des mandataires délégués pour statuer et agir au nom de ceux qu'ils représentent au mieux de leurs intérêts. Cette délégation peut être, on le sait, conçue de deux manières: ou bien il s'agit, pour les délégués, de décider en respectant au mieux les préférences de ceux qu'ils représentent; ou bien il s'agit pour eux de décider sur la base de leur jugement ce qu'ils estiment être le mieux pour les citoyens, que ce soit leur électorat ou la société dans son ensemble. Mais dans les deux cas, les représentants doivent, comme il a été indiqué plus haut, rendre compte en permanence de $c e$ qu'ils font et disent vis-à-vis des représentés ${ }^{34}$. Les décisions prises par un élu, par un parti ou par un candidat à l'élection sont susceptibles d'être contestées par les représentés: ou bien ils estiment que leurs préférences ne sont pas vraiment prises en considération par les décisions (ou propositions des représentants) ou bien ils estiment que les représentants n'ont pas, dans leur jugement, suffisamment ou adéquatement tenu compte des intérêts des représentés. Dans les deux cas, les citoyens disposent d'une base sur laquelle étayer leur contestation de la décision incriminée: soit le respect de leurs préférences, soit l'idée qu'ils se font de l'intérêt public. Et la contestation peut parfois conduire à mettre en question l'aptitude des élus, ou d'un parti, à tenir compte des préférences ou des intérêts des citoyens et à les sanctionner en ne les réélisant pas. L'élection n'est donc jamais une simple autorisation: elle implique le contrôle périodique et la «responsivité ${ }^{35}$ (responsiveness) permanente des élus. Et ce contrôle et cette «responsivité » se font sur base d'un examen public des prétentions élevées par les élus (ou les candidats à l'élection), prétentions relatives à la justesse des positions qu'ils avancent et à leurs aptitudes à gouverner. Bien entendu, l'exposé de ces prétentions peut prendre la forme d'une rhétorique et dissimuler des intérêts particuliers. Mais la dynamique électorale, le besoin d'obtenir et de garder la confiance de l'électeur oblige l'élu ou le candidat à l'élection à soumettre ses prétentions propres au jugement des citoyens.

34. L'assemblée est «responsive» (Pettit, 20I 2, I97-198).

35. Que le lecteur nous pardonne ce néologisme. Le mot «réactivité » ne nous semble pas en effet rendre pleinement compte de l'idée de «répondre à» incluse dans le mot anglais «esponsiveness». 
Il en irait autrement dans une assemblée tirée au sort où la qualification à représenter serait exclusivement conférée par le dispositif de la sélection aléatoire. L'échange entre représentants et représentés ne serait certes pas inconcevable, mais il serait dépourvu de toute force d'engagement. Le rapport entre eux est en effet d'une tout autre nature. Les représentants sont des citoyens «comme les autres» qui, compte tenu de la situation optimale d'information et de délibération dans laquelle ils sont placés, sont censés exprimer collectivement ce que seraient les opinions et la/les décision(s) de l'ensemble des citoyens s'ils pouvaient être placés dans la même situation. La représentation est "indicative" (Pettit 20I2, I99-200) non pas de l'état effectif de l'opinion ou des préférences effectives des citoyens, comme dans un sondage d'opinion, mais de ce que devrait être leur opinion ou leur décision dans une situation idéale de délibération (la définition de ce qu'est cette situation idéale pouvant du reste varier). Il s'ensuit que la légitimité de la résolution prise par une assemblée ainsi conçue est fondée sur les seules caractéristiques formelles de l'échantillon et de la situation dans laquelle il est placé.

La propriété formelle la plus souvent invoquée sera la représentativité statistique, de la composition de l'assemblée: elle est un «mini-public» (ou un mini-dèmos) dont les décisions sont censées être identiques à celles que le démos dans son ensemble prendrait pour lui-même. Bien entendu, les membres de l'assemblée sont, dans le meilleur des cas, censés s'informer et délibérer entre eux avant de décider. Mais ce qui fait la supériorité de leur délibération sur toute autre délibération, notamment celle d'une assemblée élue, ce qui donne à leur délibération son autorité, c'est qu'elle est le fait d'un échantillon statistiquement représentatif du grand public. De sorte que l'issue de la décision est censée équivaloir à celle qu'aurait pu prendre le grand public, le dèmos dans son ensemble. L'écart existant entre représentants et représentés est censé être comblé non par des interactions entre eux, mais par le fait que, en vertu des caractéristiques de la situation dans laquelle ils sont placés, les représentants - ici les citoyens tirés au sort - expriment collectivement le point de vue que les représentés adopteraient s'ils avaient l'occasion de délibérer et de décider tous ensemble.

$\mathrm{Eu}$ égard à cette fonction indicative dévolue aux mini-publics, les citoyens représentés ne disposent d'aucune base substantielle pour contester quant au fond ce qu'accomplissent, disent et décident leurs «représentants " dans ce cadre. Ce ne sont donc ni leurs aptitudes à bien gouverner ni la congruence de leur position avec celle des représentés qui les fondent à décider et à agir en leur nom, mais seulement les qualités formelles de la situation dans laquelle ils sont placés. Même si les positions prises par les membres d'un mini-public ne coïncident pas avec les convictions actuelles de leurs concitoyens, ce sont ces positions et décisions que ceux-ci auraient adoptées s'ils avaient été à la place des représentants. Ils n'ont pas non plus à contester le défaut de compétence de certains de leurs représentants 
puisque ceux-ci n'ont pas à être compétents; ou s'ils ont à l'être, c'est à titre de «citoyens ordinaires ", le tirage au sort étant le garant de leur ordinarité.

Il s'ensuit que les citoyens membres d'une assemblée tirée au sort n'ont de compte à rendre vis-à-vis des autres citoyens ni sur les positions qu'ils adoptent ni sur leurs compétences à délibérer et à décider si ce pouvoir leur a été conféré. Ils peuvent bien entendu être sommés de justifier leurs opinions vis-à-vis des autres membres de l'assemblée, mais pas vis-à-vis du grand public. Car au fond, ce qui importe n'est pas ce que chacun pense individuellement, c'est plutôt le jugement et la décision qui émane de l'exercice collectif d'une délibération dans lequel les membres de l'assemblée échangent mutuellement des arguments mais ne s'adressent pas aux citoyens.

Les seuls motifs de contestation politique des décisions d'une assemblée tirée au sort ne peuvent donc être que procéduraux: relatifs aux modalités de sélection et de délibération et au respect du mandat qui lui a été donné. Un citoyen pourrait faire valoir que l'échantillon sélectionné n'est pas vraiment représentatif ou que la délibération n'a pas été équitable et que, par conséquent la décision prise est illégitime. Mais il ne s'agirait alors que d'une mise en cause indirecte, ne touchant pas au fond des positions, et dont l'issue devrait être tranchée par des experts en statistiques ou en procédures délibératives plutôt que par des citoyens.

Cela signifie aussi que les représentants membres d'une assemblée tirée au sort n'ont pas à justifier leurs positions à l'égard de ceux qu'ils représentent. Ils sont, dans le meilleur des cas, censés délibérer entre eux afin d'adopter une position raisonnée. Ils peuvent informer le grand public de leurs positions et de leurs raisons. Mais ils n'ont pas à en rendre raison puisque c'est leur position même dans une assemblée indicative et délibérante qui leur donne un accès privilégié aux bonnes raisons. Le citoyen ordinaire non participant n'y a pas accès puisqu'il n'est pas dans ces conditions d'une délibération optimale. Au mieux, on peut l'éduquer à ces bonnes raisons.

Cela veut dire aussi que la confiance accordée par le citoyen à ceux qui le représentent dans une assemblée tirée au sort ne repose pas sur le fait qu'ils partageraient les mêmes convictions ou qu'il y aurait une forme d'engagement mutuel et de contrôle possible du respect de l'engagement, comme cela peut être le cas dans une assemblée élue. La confiance des représentés repose exclusivement sur la qualité procédurale supposée des dispositifs d'échantillonnage et de délibération (Laffont, 2015).

On pourrait dès lors se demander si les propositions d'instauration d'un parlement tiré au sort ne reproduisent pas, à leur insu, le schéma des théories élitistes de la représentation: il s'agit de confier le pouvoir de gouverner à un groupe de citoyens qui sont dans les meilleures conditions pour délibérer. Seul le tirage au sort réaliserait ces conditions. La différence avec le parlementarisme classique tiendrait à ce que la qualité de délibération ne serait pas liée aux qualités particulièrement exceptionnelles de ces citoyensreprésentants, mais plutôt à la qualité de l'échantillon statistique et à la 
procédure dans laquelle on le place. Dès lors, une telle approche négligerait l'importance et les effets que les débats informels entre citoyens dans l'espace public peuvent avoir pour la formation de la délibération politique dans les instances de décisions, singulièrement dans les assemblées représentatives.

Comme le souligne $\mathrm{Ph}$. Pettit, il existe un risque qu'une assemblée ainsi constituée se mue en "despote bienveillant» (20I2, 205). Et la possibilité pour les citoyens ou les acteurs de la société civile de prévenir ce risque se trouverait fortement réduite par le fait que le pouvoir de contrôle donné par l'élection aurait disparu et que leurs positions se verraient disqualifiées puisqu'elles n'auraient pas été produites dans de bonnes conditions de délibération. Non seulement une assemblée tirée au sort ne serait pas tenue de se soucier des opinions des citoyens qui n'y participent pas, mais de plus elle n'inciterait pas ceux-ci à développer leur propre jugement politique. Elle leur demanderait une confiance, "une déférence aveugle» (Laffont, 20 I 5) à l'égard du jugement de certains d'entre eux, qui leur ressemblent - et parce qu'ils leur ressemblent - et que le sort aurait placés dans des conditions de délibération supposées optimales.

En revanche, la dynamique électorale peut, quant à elle, contribuer à la formation raisonnée du jugement politique des citoyens, du plus grand nombre de citoyens. Elle sollicite les opinions de tous les citoyens et par son caractère concurrentiel incite à une confrontation de ces opinions. Émettre un vote, c'est en effet aussi reconnaître, même si c'est souvent confusément, une prétention à la validité à une opinion politique émise par un acteur.

On pourrait objecter à ce qui précède que ce qui est fondamentalement important, c'est que les décisions prises soient les meilleures possibles, les mieux informées et les mieux raisonnées. Or, de ce point de vue, les performances épistémiques d'une délibération au sein d'une assemblée tirée au sort pourraient sembler nettement supérieures à celles de la délibération d'une assemblée élue.

\subsection{La qualité épistémique des décisions}

Les procédures de décision publiques ne doivent pas seulement être inclusives et contrôlables par les citoyens: elles devraient aussi être configurées de manière à maximiser l'occurrence de décisions justes, informées et raisonnées ou, en tout cas, reconnues comme telles ${ }^{36}$. Selon les théories délibératives, cela suppose que les décisions soient prises à l'issue d'un échange public d'arguments tant au sein des espaces publics informels, où se forment librement les opinions, que dans les procédures instituées qui doivent être délibératives et ouvertes aux débats publics informels. De ce point de vue, le mode privilégié de désignation de l'assemblée - élection ou tirage au sort

36. Comme nous l'avons mentionné plus haut (note 20), nous laissons ici de côté la question de savoir si l'une de ces conditions est plus importante que l'autre. 
— devrait être celui qui favorise au mieux les conditions d'une délibération raisonnée et inclusive génératrice de qualité épistémique dans la décision.

Il semble, prima facie, que l'élection au suffrage universel favorise davantage la rhétorique plébiscitaire ${ }^{37}$ et le marchandage plutôt qu'une formation raisonnée des opinions et des décisions. Les représentants seraient avant tout mûs par leur intérêt à se faire réélire et prêts pour ce faire à user de moyens rhétoriques douteux, à travestir les faits, à dire à l'électeur ce qu'il souhaite entendre, à pratiquer la sophistique ou à agiter les émotions. Et lorsqu'il s'agit de décider collectivement, de constituer une majorité, c'est le rapport de force et le marchandage qui prévaudraient plutôt que la recherche coopérative de la meilleure solution. En revanche, des assemblées ou des jurys sélectionnés sur une base aléatoire, dûment informés et soumis à l'obligation de délibérer collectivement et de prendre une décision motivée produiraient des performances épistémiques nettement supérieures à celle d'assemblées élues, et ce, pour plusieurs raisons.

- Les membres d'une assemblée tirés au sort n’ont pas, par définition, d'intérêt à se faire réélire. Leur carrière et leur réputation ne sont pas en jeu. Ils sont donc davantage susceptibles d'impartialité et d'objectivité dans les jugements qu'ils posent. Leur inexpérience (incompétence) relative par rapport à des professionnels de la politique peut aisément être compensée par des moyens d'information et de formation appropriés. Il semble d'ailleurs que les compétences politiques spécifiques des élus soient davantage liées à la stratégie politique qu'au contenu des dossiers qu'ils ont à traiter.

- La sélection par tirage au sort favorise une diversité sociale plus grande que dans une assemblée élue, qui est certainement plus homogène socialement sur le plan du recrutement. Cette diversité sociale induit une diversité de perspectives et de sources d'informations, ce qui contribue à la qualité des jugements qui fondent les décisions.

- Enfin, les conditions internes d'une assemblée tirée au sort favorisent des échanges entre les participants qui sont plus proches de ce que l'on attend d'une vraie délibération. De fait, les risques de la rhétorique plébiscitaire sont réduits, de même que ceux du marchandage.

Il faut cependant remarquer que le recours à l'élection des assemblées parlementaires n'est pas dépourvu d'atouts épistémiques au regard de la désignation par tirage au sort.

37. Simone Chambers $(2009,338)$ définit la rhétorique plébiscitaire comme suit: "Plebiscitary rhetoric, as I have been using the term, refers to speech that is concerned first and foremost with gaining support for a proposition and only secondarily with the merits of the arguments or persuasion for that matter. Plebiscitary rhetoric deploys speech strategically for the purposes of winning”. 
- Il est vraisemblable que les compétences à débattre, à user de la rhétorique mais aussi à la déjouer sont plus également distribuées dans une assemblée élue qu'elles ne le seraient dans une assemblée tirée au sort. Dans un parlement élu, les députés jouent à armes à peu près égales. Il y a bien sûr des néophytes, mais ceux-ci sont normalement soutenus et formés par leur parti de sorte que leur relatif désavantage peut être assez rapidement compensé — ou parfois utilisé — par le groupe parlementaire auquel ils appartiennent. En revanche, en favorisant la diversité sociale, le mode de sélection aléatoire induit aussi une plus grande inégalité de niveaux de formation et dans les compétences politiques utiles à l'exercice du mandat. Cette inégalité plus forte entre mandataires serait aussi plus difficile à compenser. En tout cas, l'absence de parti politique, inhérente à la sélection aléatoire, prive les assemblées composées sur cette base d'un moyen d'intégration et de formation de ses membres.

- Les discours, les délibérations et les décisions prises dans une assemblée élue sont en permanence soumis à la vigilance et à la critique non seulement des citoyens mais des associations et organisations de la société civile. Un élu peut user de la rhétorique plébiscitaire. Mais celle-ci peut aussi être déjouée par le discours d'un élu d'un autre bord et surtout par le contrôle permanent que les organisations de la société civile et les médias exercent sur eux. Cette conflictualité interne et ce contrôle permanent, s'ils ne sont pas infaillibles ni dépourvus de visées stratégiques, possèdent toutefois des vertus épistémiques. Même si un élu défend un intérêt particulier (le sien propre ou celui d'un groupe), il a avantage à le défendre en parlant le langage de l'intérêt public et en évitant de mentir. À défaut, sa partialité ou sa perfidie risquent d'être mises au jour et dénoncées par ses adversaires politiques ou par des citoyens ou des acteurs attentifs. Bien entendu, ce risque peut amener l'élu à travestir, sous les dehors acceptables d'un argument de bien public, la promotion d'un intérêt particulier. On peut néanmoins faire l'hypothèse, à la suite de J. Elster (I994), que cette hypocrisie a une "vertu civilisatrice». Même si elle est secrètement motivée par un intérêt électoraliste, l'expression publique d'une opinion politique oblige celui qui la tient à invoquer des raisons acceptables par le plus grand nombre et à conserver une relative cohérence vis-à-vis de cellesci, ce qui peut, en retour, le conduire à amender sa position initiale pour la rendre plus proche de l'intérêt public.

Dans une assemblée tirée au sort on ne retrouverait pas cette dynamique d'affrontement systématique qui peut être le ferment de la critique mutuelle des positions ${ }^{38}$. De plus, le contrôle externe serait aussi moins

38. Sur l'importance de la dynamique du contradictoire dans la délibération démocratique, voir Manin, 20II. 
généralisé. Les positions défendues par les membres d'une assemblée tirée au sort n'attirent pas la suspicion permanente et la forte légitimité qui est attribuée au point de vue des citoyens ordinaires délibérant en leur sein et pouvant conduire à désarmer et disqualifier les positions des organisations de la société civile, suspectées, quant à elles, de défendre un intérêt sectionnel, donc partial. Paradoxalement la valorisation de la participation des citoyens ordinaires, sous la modalité de la sélection aléatoire, peut ainsi contribuer à éroder la force de la critique sociale, laquelle contribue souvent à l'émergence de nouvelles politiques plus justes.

- Enfin, il ne faut pas négliger les vertus généralisantes de la dynamique électorale elle-même. J'ai évoqué, au point précédent, le fait que la conflictualité politique pouvait contribuer à la mise à l'épreuve des positions. Il faut aussi souligner que la dynamique électorale contraint à une montée en généralité (Urbinati 2006). Les candidats à l'élection doivent s'adresser à l'audience la plus large possible et donc prendre en considération une diversité d'intérêts et de points de vue. De plus, lors d'une élection, tous les enjeux, toutes les questions sont susceptibles d'être mis sur la table par les citoyens et par les acteurs sociaux et politiques, pour peu qu'ils s'engagent collectivement. Enfin, la dynamique électorale et le fait que les représentants soient élus pour la durée d'une mandature induit les candidats à produire une hiérarchisation des questions et des objectifs et à veiller à leur articulation dans un programme politique soumis publiquement au jugement de tous les électeurs en un lieu et un moment unique, commun. Il est rare qu'un parti n'ait qu'un seul objectif à son programme. Et si c'est le cas, il est alors tenu de justifier cette priorité, qui doit trouver grâce aux yeux du plus grand nombre d'électeurs. De ce fait, la «montée en généralité » ne se situe pas seulement du côté des partis et des candidats: en le confrontant à une pluralité d'enjeux et de points de vue, l'élection invite l'électeur à adopter un point de vue plus général que celui qui serait lié à sa préoccupation immédiate.

Les observations qui précèdent ne reposent pas sur une vision idéalisée et naïve de la compétition électorale, à laquelle on pourrait aisément opposer l'irrationalité de l'électeur et le cynisme des acteurs politiques. Il s'agissait simplement d'identifier des potentialités trop souvent inexploitées. Leurs bénéfices épistémiques sont certes limités et doivent toujours être contrebalancés avec ceux que produirait une sélection des mandataires par tirage au sort. Mais ils sont aussi suffisamment spécifiques pour que, même d'un point de vue strictement épistémique, nous ne renoncions pas totalement à l'élection pour nous en remettre exclusivement au hasard à l'heure de désigner des gouvernants. 


\section{Conclusions}

La confrontation entre les vertus respectives du tirage au sort et de l'élection nous montre que cette dernière possède des vertus démocratiques que n'a pas la première lorsqu'il s'agit de désigner les membres d'une assemblée gouvernante. Cela ne disqualifie certes pas le recours au tirage au sort lorsqu'il s'agit d'associer des citoyens à la prise de décision publique. Mais cela montre que le remplacement pur et simple des scrutins par la sélection aléatoire n'est certainement pas souhaitable. Car les élections ont des qualités propres qui les rendent essentielles dans une démocratie. Elles assurent une imputation de responsabilité pour les décisions; elles permettent une forme de participation politique égalitaire, individualisée et accessible sans autre condition que celle d'être citoyens, tout en contribuant à la socialisation politique et à la reconnaissance mutuelle de ceux-ci; les élections instaurent un espace d'interaction entre gouvernants et gouvernés qui rend possible la contestation et le contrôle par ceux-ci des décisions prises par ceux-là; les élections favorisent l'âpreté critique du débat public et sa montée en généralité: elles peuvent ainsi contribuer à la qualité épistémique des délibérations. Si les élections ne sont pas l'essence même de la démocratie, elles possèdent des vertus essentielles à la démocratie.

En identifiant ces vertus, je n'entends pas nier les difficultés ni minimiser les défis auxquels les formes contemporaines de la démocratie électorale sont confrontées. Je n'ai pas cette naïveté. Mais les quelques réflexions que j'ai proposées offrent des critères nous permettant de comprendre ce qui ne va pas et d'essayer d'imaginer des solutions susceptibles d'améliorer nos systèmes électoraux non seulement dans leur fonctionnement interne mais aussi, et surtout, dans leur articulation avec l'espace public. Il est possible que ces solutions intègrent, parmi d'autres innovations, le recours au tirage au sort de citoyens dans des instances délibérantes dotées d'un pouvoir de codécision. Cette pratique semble en effet posséder quelques bénéfices que la littérature sur la question met aujourd'hui en avant, même si cette solution doit encore être éprouvée. Il n'est ainsi pas exclu que l'on puisse articuler, par exemple, dans un bicaméralisme renouvelé, élection et tirage au sort. Mon propos n'était cependant pas ici d'examiner les vertus démocratiques du tirage au sort et de son éventuelle articulation avec les pratiques électorales. Il visait plutôt à montrer que ces dernières demeurent essentielles et ne pourraient, à plusieurs égards, être purement et simplement remplacées par le tirage au sort. Je voulais ainsi faire ressortir un point trop négligé par les réflexions normatives actuelles sur la démocratie, qu'elles émanent des acteurs ou des milieux académiques. C'est à partir de la démocratie électorale, et non contre elle ou en dépit d'elle, qu'il faut s'efforcer de régénérer la démocratie dans nos sociétés de masse. 


\section{Références}

Bacqué, Marie-Hélène, Henri Rey et Yves Sintomer. «La démocratie participative, un nouveau paradigme de l'action publique?" in Marie-Hélène Bacqué et al., Gestion de proximité et démocratie participative, Paris, La Découverte, 2006, p. 9-46.

Bernardi, Bruno. Qu'est-ce qu'une décision politique? Paris, Vrin, 2013.

Blondiaux, Loïc. Le nouvel esprit de la démocratie: Actualité de la démocratie participative, Paris, Seuil (La république des idées), 2008.

Bohman, James \& William Regh (dir.). Deliberative Democracy. Essays on Reason and Politics, Cambridge (Mass.)/London, The MIT Press, I999.

Bovens, Mark. "Analysing and Assessing Accountability: A Conceptual Framework", European Law Journal, I3 (4), 2007, p. 447-468.

Chambers, Simone. "Rhetoric and the Public Sphere: Has Deliberative Democracy Abandoned Mass Democracy?", Political Theory, vol. 37, ${ }^{\circ}$ 3, 2009, p. 323350.

Cohen, Joshua. "Deliberation and Democratic Legitimacy», in Bohman \& Regh, I999, p. 67-9I.

Cossart, Paula. Le meeting politique. De la délibération à la manifestation (I868I939), Rennes, PUR, 20 Iо.

Cristin, Olivier. Vox populi. Une histoire du vote avant le suffrage universel, Paris, Seuil, 2OI4.

Deloye, Yves et Olivier Ihl. L'acte de vote, Paris, Presses de Sciences Po, 2008.

Elster, Jon. "Argumenter et négocier dans deux assemblées constitutantes ", Revue française de science politique, vol. 44, $\mathrm{n}^{\circ} 2$, I994, p. I87-256.

(dir.) Deliberative Democracy, Cambridge, Cambridge University Press, I 998.

Faucher, Florence et Colin Hay. «Les rituels de vote en France et au Royaume-Uni », Revue française de science politique, vol. $65, \mathrm{n}^{\circ} 2,20 \mathrm{I} 5, \mathrm{p}$. 2I3-236.

Gaxie, Daniel. "Cognitions, auto-habilitation et pouvoirs des "citoyens" ", Revue française de science politique, 57 (6), 2007, p. 737-757.

Girard, Charles. "La règle de majorité en démocratie: équité ou vérité ?", Raisons politiques, $\mathrm{n}^{\circ} 53,20 \mathrm{I} 4$, p. I07-I 37 .

Girard, Charles et Alice Le Goff (dir.). La démocratie délibérative. Anthologie de textes fondamentaux, réunis par C. Girard et A. Le Goff, Paris, Hermann, 2010.

Gonzalez-Ricoy Iñigo \& Axel Gosseries (dir.). Institutions for Future Generations, Oxford University Press, à paraître, 2017.

Goodin, Robert. "Enfranchising All Affected Interests, and Its Alternatives », Philosophy and Public Affairs, 35 (I), 2007, p. 40-68.

Guizot, François. I826. "Élections" [Encyclopédie progressive, I826], Discours académiques, Paris Didier, I86I.

Hansen, Mogens H. La démocratie athénienne à l'époque de Démosthène. Structure, principes et idéologie, trad. S. Bardet avec le concours de Ph. Gauthier, Paris, Tallandier (Texto), 2009.

Ihl, Olivier. Le vote, Paris, Montchrestien, 2000.

Lafont, Cristina. "Deliberation, Participation, and Democratic Legitimacy: Should Deliberative Mini-publics Shape Public Policy? ", The Journal of Political Philosophy, vol. 23, $\mathrm{n}^{\circ}$ I, $20 \mathrm{I} 5$, p. 40-63. 
Landemore, Helene. «Deliberation, Cognitive Diversity, and Democratic Inclusiveness: An Epistemic Argument for the Random Selection of Representatives. » Synthese I90 (7), 2013, p. I209-I23I.

Leydet, Dominique (dir.). La démocratie délibérative, Philosophiques, vol. 29, $\mathrm{n}^{\circ} 2$, 2002.

Lopez-Guerra, Claudio. Democracy and Disenfranchisement. The Morality of Electoral Exclusions, Oxford, Oxford University Press, 20I4.

MacCormick, John P. Machiavellian Democracy, Cambridge University Press, 20 I I.

Mair, Peter. Ruling the Void: the Hollowing of Western Democracy, Londres, Verso, 2013.

Manin, Bernard. «Volonté générale ou délibération? Esquisse d'une théorie de la délibération politique ", Le Débat, $\mathrm{n}^{\circ} 33, \mathrm{I} 985$, p. 72-93.

—. Principes du gouvernement représentatif, Paris, Flammarion, I995.

- "Comment promouvoir la délibération démocratique? Priorité du débat contradictoire sur la discussion ", Raisons politiques, 42, 20I I, p. 83-I I3.

Mansbridge, Jane e.a. "The Place of Self-Interest and the Role of Power in Deliberative Democracy", The Journal of Political Philosophy, vol. I8, $\mathrm{n}^{\circ}$ I, 2010, p. 64-100.

Marx, Karl. Critique du droit politique hégélien, Paris, Ed. sociales, I972.

Meyer, Norra. Sociologie des comportements politiques, Paris, A. Colin, 2010.

Norris, Pippa. Democratic Phoenix: Reinventing Political Activism, New York, Cambridge University Press, 2002.

Papadopoulos, Yannis. "Accountability and Multi-level Governance: More Accountability, Less Democracy?", West European Politics, vol. 33, n 5, 2010, p. IO30-I049.

- - "On the Embeddedness of Deliberative Systems: Why Elitist Innovations Matter More» in Deliberative Systems. Deliberative Democracy at the Large Scale, J. Parkinson \& J. Mansbridge (eds), Cambridge, Cambridge University Press, 20I 2, p. I25-I 50.

Parkinson, John. Deliberating in the Real World. Problems of Legitimacy in Deliberative Democracy, Oxford, Oxford University Press, 2006.

Pettit, Philip. On the People's Terms. A Republican Theory and Model of Democracy, Cambridge, Cambridge University Press, 20 г 2.

Pourtois, Hervé. "Mini-publics et démocratie délibérative", Politique et Sociétés, vol. $32, \mathrm{n}^{\circ} \mathrm{I}, 20 \mathrm{I} 3$, p. $2 \mathrm{I}-4 \mathrm{I}$.

Przeworski, Adam, Susan C. Stokes \& Bernard Manin (eds.). Democracy, Accountability, and Representation, Cambridge, Cambridge University Press, I999.

Risse, Mathias. "Arguing for Majority Rule», The Journal of Political Philosophy, vol. I $2, \mathrm{n}^{\circ} \mathrm{I}, 2004$, p. 4I-64.

Rosanvallon, Pierre. Le peuple introuvable. Histoire de la représentation démocratique en France, Paris, Gallimard, I998.

—. La légitimité démocratique. Impartialité, réflexivité, proximité, Paris, Seuil, 2008.

Saffon, Maria Paula \& Nadia Urbinati. «Procedural Democracy, the Bulwark of Equal Liberty ", Political Theory, 4I/3, 20I3, p. 44I-48I.

Saunders, Ben. "Defining the Demos", Politics, Philosophy \& Economics, I I (3) 280-3OI, 20II.

Sintomer, Yves. Petite histoire de l'expérimentation démocratique. Tirage au sort et politique d'Athènes à nos jours, Paris, La Découverte, coll. "Poche/Essais », $201 \mathrm{I}, 296 \mathrm{p}$. 
- «Tirage au sort et démocratie délibérative. Une piste pour renouveler la politique au XXI ${ }^{\mathrm{e}}$ siècle? ", Vie des idées, 5 juin 20I2. [http://www.laviedesidees.fr/ Tirage-au-sort-et-democratie-deliberative.html]

Sintomer, Yves et Julien Talpin (dir.). Démocratie délibérative, Raisons politiques, $\mathrm{n}^{\circ} 42$, $20 \mathrm{II}$.

Sutherland, Keith. A People's Parliament: A (Revised) Blueprint for a Very English Revolution, Exeter, Imprint Inc, 2008.

Thompson, Dennis F. Just Elections: Creating a Fair Electoral Process in the United States, Chicago, University of Chicago Press, 2002.

Urbinati, Nadia. Representative Democracy: Principles and Genealogy, Chicago, The University of Chicago Press, 2006.

Van Reybrouck, David. Contre les élections, Arles, Actes Sud, coll. «Babel/essai» $\left(\mathrm{n}^{\circ} \mathrm{I} 23 \mathrm{I}\right), 20 \mathrm{I} 2$.

Weale, Albert. Democracy, $2^{\mathrm{e}}$ éd., New York, Palgrave MacMillan, 2007.

White, Jonathan \& Lea Ypi. «On Partisan Political Justification", American Political Science Review, I05/2, 20 I I, p. 38 I-396. 